\title{
Prototipo inalámbrico para carga de vehículos eléctricos
}

\author{
Martínez, Andrés \\ Universidad Tecnológica de Panamá, Facultad de Ingeniería Eléctrica \\ Panamá, Panamá \\ andres.martinez@utp.ac.pa \\ González, Christian \\ Universidad Tecnológica de Panamá, Facultad de Ingeniería Eléctrica \\ Panamá, Panamá \\ christian.gonzalez4@utp.ac.pa \\ Von Chong, Alejandro \\ Universidad Tecnológica de Panamá, Facultad de Ingeniería Eléctrica \\ Panamá, Panamá \\ alejandro.von@utp.ac.pa
}

\begin{abstract}
Wireless power transfer (WPT) is an attractive solution for charging electric vehicles, the demand for which will increase with the National Electric Mobility Strategy in Panama. However, a factor that negatively affects it is the misalignment between the transmitter and receiver coils, as it causes a decrease in the transferred power. In order to mitigate this problem, the design and construction of a wireless charger prototype is presented to characterize the effects of misalignment, using a half-bridge inverter and coils with seriesseries compensation. A microcontroller is used to supply and monitor high frequency signals. A resistor was used as a load. The results show that, by operating the system at the resonant frequency, power transfer is maximized and there is no phase shift between the voltage and current waves. On the other hand, by creating controlled misalignments in the coils, there is a change in the resonance frequency of the system and a proportional phase shift in the aforementioned waves is originated.
\end{abstract}

Keywords: Magnetic coupling, misalignment, phase shift, resonant frequency, wireless power transfer. 


\section{Resumen}

La transferencia inalámbrica de potencia (WPT) es una solución atractiva para cargar vehículos eléctricos, cuya demanda aumentará con la Estrategia Nacional de Movilidad Eléctrica en Panamá. Sin embargo, un factor que la afecta negativamente es el desalineamiento entre las bobinas transmisora y receptora, pues provoca la disminución de la potencia transferida. Buscando mitigar este problema, se presenta el diseño y construcción de un prototipo de cargador inalámbrico para caracterizar los efectos del desalineamiento, empleando un inversor medio puente y bobinas con compensación serie-serie. Un microcontrolador es utilizado para suplir y monitorear señales de alta frecuencia. Se utilizó una resistencia como carga. Los resultados muestran que, al operar el sistema a la frecuencia de resonancia, se maximiza la transferencia de potencia y no existe desfase entre las ondas de voltaje y corriente. Por otro lado, al crear desalineamientos controlados en las bobinas, hay un cambio en la frecuencia de resonancia del sistema y se origina un desfase proporcional en las ondas mencionadas.

Palabras claves: Acoplamiento magnético, desalineamiento, desfase, frecuencia de resonancia, transferencia inalámbrica de potencia.

\section{INTRODUCCIÓN}

En décadas recientes, el auto eléctrico ha surgido como una alternativa para subsanar las consecuencias negativas derivadas de los vehículos de combustión interna. De hecho, su mercado presenta un crecimiento exponencial: en el 2010, apenas había unos 17,000 vehículos eléctricos, cifra que para el 2020 aumentó a 7.2 millones, según la International Energy Agency [1]. No obstante, este crecimiento está limitado por la cantidad de estaciones de carga disponibles. En Panamá, con la implementación de la Estrategia Nacional de Movilidad Eléctrica, se espera un aumento en la demanda de vehículos eléctricos y, consecuentemente, de estaciones de carga [2]. Debido a que los sistemas de carga tienen un impacto en la penetración de la movilidad eléctrica, es una necesidad conocer cuáles son los desafíos que enfrenta esta industria y la forma en la que evoluciona.

Dentro de las tecnologías de carga, se encuentra la transferencia inalámbrica de potencia (WPT por sus siglas en inglés), la cual se compone de un dispositivo transmisor, que transforma la energía eléctrica en un campo electromagnético, y de uno o más dispositivos receptores, los cuales convierten la potencia/energía presente en este campo para la recarga de la batería del vehículo. Desde el punto de vista funcional, es evidente 
que la WPT es útil para estas aplicaciones, sin embargo, los diseños aún presentan retos como operar eficientemente en condiciones de desalineamiento físico entre el transmisor y el receptor.

En la investigación realizada, se diseñó y construyó un prototipo de cargador inalámbrico de potencia con el objetivo de caracterizar los efectos de operarlo bajo desalineamientos verticales controlados, empleando para esto un sistema de monitoreo de variables en la antena transmisora y en la carga. El prototipo, hecho con topología serie-serie, convierte la alimentación de una fuente DC en AC a través de un inversor medio puente de MOSFETs, que a su vez recibe las señales de control de un microcontrolador. El inversor alimenta a la antena transmisora, que transfiere inalámbricamente la potencia hacia la antena receptora.

Este artículo inicialmente describe los materiales y la metodología utilizados para la construcción del prototipo. Luego, se presentan y discuten los resultados y finalmente, las conclusiones obtenidas en el proyecto.

\section{MÉTODO}

\section{A. Sistema para transferir inalámbricamente la potencia}

Las bobinas, fabricadas por Würth Elektronik (Hohenlohe, Alemania), son un producto especial para aplicaciones de carga inalámbrica, ya que cumplen con el estándar Qi del Wireless Power Consortium [3]. Adicionalmente, reposan sobre un material de alta permeabilidad magnética (NiZn), lo cual aumenta el acoplamiento y, consecuentemente, la transferencia de potencia. Las bobinas fueron colocadas dentro de acrílicos para manejarlas más convenientemente. Ni este material ni el de los tornillos es magnético.

Los capacitores en ambas antenas son cerámicos. Tienen el objetivo de compensar la capacitancia parásita e inherente de las bobinas.

Por lo general, estos sistemas operan a altas frecuencias (varios cientos de kilohertz). Esto se debe a que la eficiencia del acoplamiento magnético es directamente proporcional a los factores de calidad de las bobinas, y estos a su vez son proporcionales a la frecuencia [4]. Además, una condición deseable es que se opere a la frecuencia de resonancia, ya que se maximiza la potencia transferida [5].

\section{B. Diseño y construcción del inversor medio puente}

Su función principal es transformar los 12 VDC de la fuente DC a un voltaje AC de alta frecuencia, que es la alimentación de la antena transmisora. Esto se logra con la conmutación de dos MOSFET IRF510 [6], cuyas compuertas son activadas con un driver IR2113 [7]. Este driver recibe dos señales de control PWM, una directamente de un microcontrolador Teensy 4.1 [8] y otra invertida por una compuerta NOR SN74LS02N [9]. Por las características de 
este inversor (medio puente), su salida es una onda cuadrada de voltaje que oscila entre 6 $\mathrm{V} y-6 \mathrm{~V}$, y la corriente tiene un comportamiento sinusoidal.

\section{Diseño y construcción del sistema de medición de desfase}

Este sistema está conformado por dos amplificadores operacionales LM7171 [10], especiales para altas frecuencias (slew rate de $4100 \mathrm{~V} / \mu \mathrm{s}$ ), y una compuerta XOR modelo CD4070BE [11]. Los LM7171 se encargan de muestrear la onda de corriente en el resistor , transformarla a una onda cuadrada y enviarla a la compuerta XOR. La onda de voltaje se envía directamente a la XOR. Esta última compara ambas señales y genera un pulso cuyo ancho indica la cantidad de segundos que la onda de corriente adelanta o atrasa a la de voltaje. Un diodo Zener es necesario para la reducción del nivel de voltaje original del pulso a uno de nivel lógico y soportable por el microcontrolador. El ancho en microsegundos y la frecuencia en $\mathrm{kHz}$ son leídos por la tarjeta Teensy 4.1 y, posteriormente, esta última calcula el ángulo de desfase con la Ecuación 1.

\section{$\theta\left[^{\circ}\right]=\left(T_{d}[\mu s] / 1000000\right) \cdot 360^{\circ} \cdot f[k H z\rceil \cdot 1000$}

\section{Implementación del prototipo completo}

En la Fig. 1 se muestra el esquemático del prototipo completo que se implementó. En la Tabla 1 se muestran la notación y el valor de los parámetros de cada uno de los componentes.

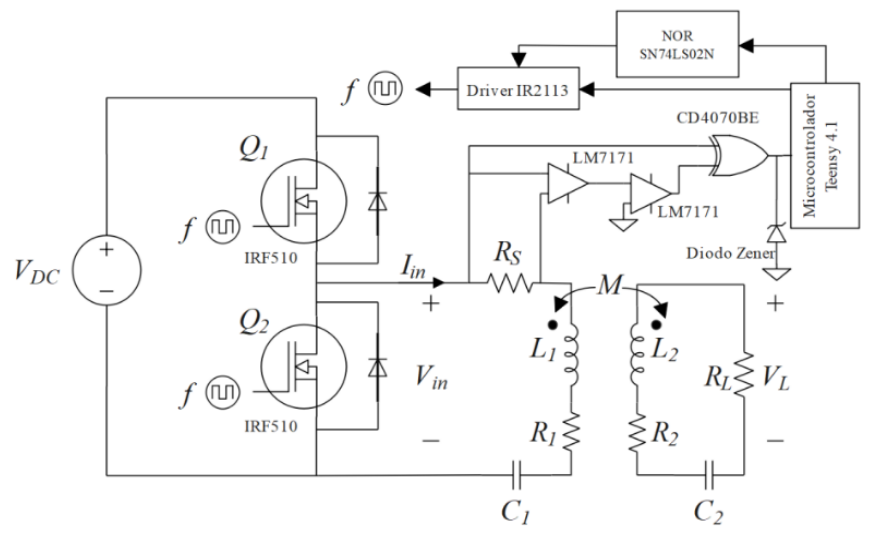

Fig 1. Esquemático completo del prototipo de cargador inalámbrico. 
Tabla 1. Notación y valores de los parámetros del protitipo.

\begin{tabular}{lll}
\hline Parámetro & Notación & Valor \\
\hline Voltaje de entrada DC & $V_{D C}$ & $12 \mathrm{~V}$ \\
Autoinductancia de la bobina transmisora & $L_{1}$ & $24 \mu \mathrm{H}$ \\
Autoinductancia de la bobina receptora & $L_{2}$ & $24 \mu \mathrm{H}$ \\
Resistencia interna de la bobina transmisora & $R_{1}$ & $0,07 \Omega$ \\
Resistencia interna de la bobina receptora & $R_{2}$ & $0,07 \Omega$ \\
Capacitor de compensación antena transmisora & $C_{1}$ & $200 \mathrm{nF}$ \\
Capacitor de compensación antena receptora & $C_{2}$ & $200 \mathrm{nF}$ \\
Resistor en derivación & $R_{S}$ & $1 \Omega$ \\
Resistor de carga & $R_{L}$ & $1 \mathrm{k} \Omega$ \\
Frecuencia de operación & $f$ & $65-90 \mathrm{kHz}$ \\
\hline
\end{tabular}

La Ecuación 2 fue utilizada para calcular la frecuencia de resonancia del sistema.

$$
f=\frac{1}{2 \pi \sqrt{24 \mu H \cdot 200 n F}}=72.6 \mathrm{kHz}
$$

\section{E. Sistema de desalineamiento vertical de las bobinas}

Para caracterizar los cambios en el comportamiento del sistema ante el aumento del desalineamiento vertical $z$ entre las bobinas, se insertaron láminas de acrílico, con un espesor de $4.25 \mathrm{~mm}$ cada una, entre ambas bobinas. Se utilizó el sistema de medición de desfase para obtener la frecuencia de resonancia y se realizaron mediciones del voltaje en la carga. Las condiciones para evaluar fueron colocándole desde 2 acrílicos hasta 5 acrílicos, es decir, desde $z=8.5 \mathrm{~mm}$ hasta $z=21.25 \mathrm{~mm}$ con incrementos de $4.25 \mathrm{~mm}$.

\section{RESULTADOS}

\section{A. Sistema de medición de desfase}

Utilizando la Teensy 4.1 y el software de Arduino, se logró calcular el ángulo de desfase entre las ondas de voltaje y corriente, ilustradas en la Fig. 2, leyendo el ancho del pulso obtenido de la compuerta XOR (Fig. 3). Para estas mediciones se realizó un barrido de frecuencia de operación con el microcontrolador en un rango de $65-90 \mathrm{kHz}$. Se pudo observar que a medida que la frecuencia de operación se acercaba a la de resonancia, el ancho del pulso disminuía hasta desaparecer y el cálculo del ángulo de desfase realizado 
por la Teensy 4.1 se aproximaba a cero. Una vez se superaba el valor de la frecuencia de resonancia, el ancho de pulso volvía aumentar su grosor, indicando que el desfase entre las ondas de voltaje y corriente también aumentaba, como también lo mostró el ángulo calculado por la Teensy 4.1.

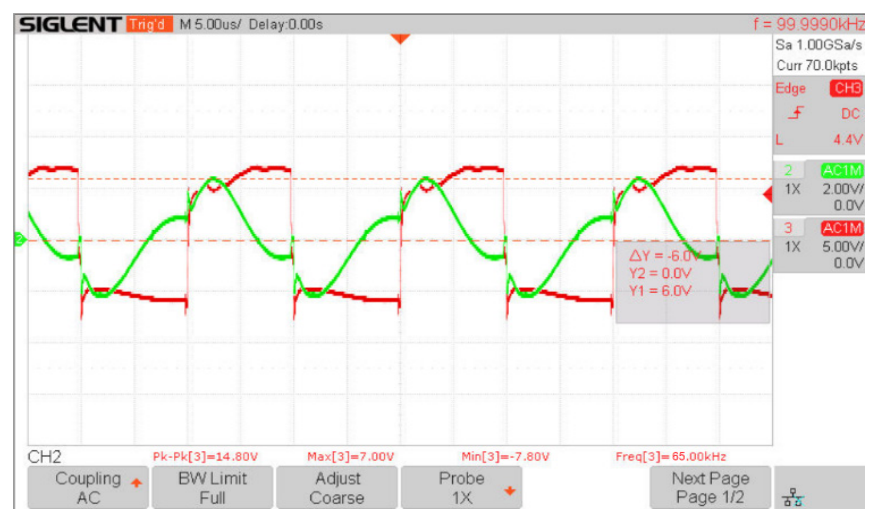

Fig 2. Ondas de voltaje (rojo) y corriente (verde).

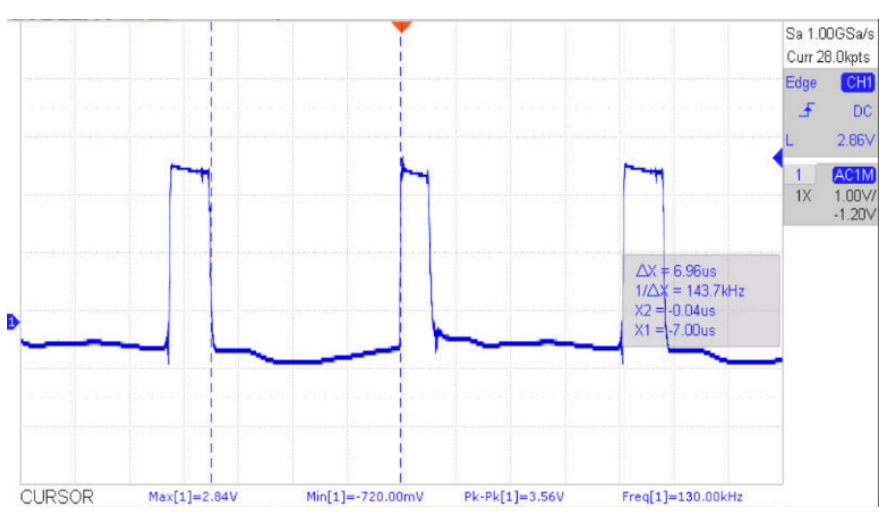

Fig 3. Pulso a la salida de la compuerta XOR.

\section{B. Voltaje en la carga}

Se observó que para diferentes desalineamientos verticales z en las bobinas, la frecuencia a la que se transfería la máxima cantidad de potencia varió. A continuación, en la Fig 4, se muestra la característica voltaje-frecuencia con diferente número de láminas entre bobinas. Se observa como a medida que aumenta el desalineamiento vertical, la frecuencia a la que se transmite el máximo voltaje a la carga es mayor. Adicionalmente, puede observarse que entre mayor es el desalineamiento entre las bobinas, menor voltaje (y potencia) se le transfiere a la carga. Estos fenómenos son explicados por la debilitación del acoplamiento magnético entre las bobinas.

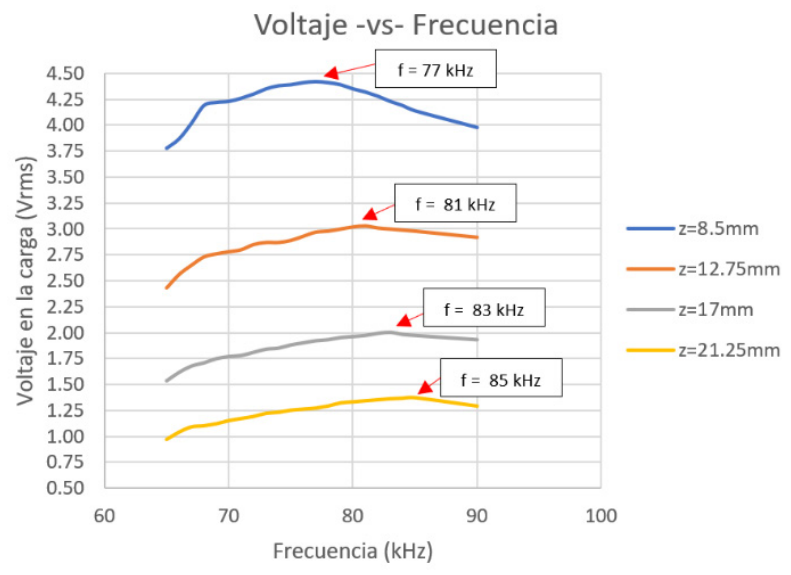

Fig 4. Característica de Voltaje - Frecuencia de la carga. 


\section{CONCLUSIONES}

Se diseñó y construyó exitosamente un prototipo de cargador WPT con topología serieserie, haciendo un aporte significativo al especificar, detallar y justificar cada componente utilizado y explicar el diseño de las diferentes etapas del proyecto con profundidad. Además, se caracterizaron los efectos del desalineamiento. Los resultados muestran que la frecuencia de resonancia con el espaciamiento vertical mínimo es cercana a la frecuencia de resonancia calculada teóricamente. Por otro lado, se observó que al aumentar el desalineamiento vertical $z$ entre las bobinas, se requiere operar con una frecuencia más elevada para alcanzar la resonancia, como se muestra en la Fig. 4. De este resultado también se concluye que se transfiere menor potencia a la carga al aumentar la separación vertical. Además, se construyó un sistema de medición de desfase entre la onda de voltaje y corriente en la antena transmisora, indicador directo del estado de resonancia en el sistema.

\section{Referencias}

[1] I. E. A. «Global EV Outlook 2020» 2020.

[2] M. Rebolledo, «Estrategia Nacional de Movilidad Eléctrica de Panamá», ONU Ambiente, Panamá, 2019.

[3] R. Narayanan, «Wireless Power Charging Coils Changing Considerations», Würth Elektronik Application Notes, 2015.

[4] P. Wiener, Analysis and Benefits of GaN in High Frequency WPT Applications. 2021.

[5] A. Treviño, J. González, J. Aguado, «Wireless Power Transfer for Electric Vehicles: Foundations and Design Approach», Springer, 2020.

[6] VISHAY, "IRF510, SiHF510 Power MOSFET," IRF510 Datasheet.

[7] International Rectifier, "IR2113 High and low side driver," IR2113 Datasheet, pp. 61-74.

[8] PJRC, "Teensy ${ }^{\circledR} 4.1$ Development Board." https://www.pjrc.com/store/teensy41.html. Consultado: $7 / 7 / 2021$.

[9] Texas Instruments, "QUADRUPLE 2-INPUT POSITIVE-NOR GATES," SN74LSO2 Datasheet, no. 1988, 1983.

[10] Texas Instruments, "LM7171 Very High Speed, High Output Current, Voltage Feedback Amplifier," LM7171 Datasheet, 2014.

[11] Texas Instruments, "CD4070B, CD4077B CMOS Quad Exclusive-OR and Exclusive-NOR Gate," CD4070BE Datasheet, no. January 1998, 2003.

\section{Autorización y Licencia CC}

Los autores autorizan a APANAC XVIII a publicar el artículo en las actas de la conferencia en Acceso Abierto (Open Access) en diversos formatos digitales (PDF, HTML, EPUB) e integrarlos en diversas plataformas online como repositorios y bases de datos bajo la licencia Attribution-NonCommercialShareAlike 4.0 International (CC BY-NC-SA 4.0) https://creativecommons.org/licenses/by-nc-sa/4.0/. Ni APANAC XVIII ni los editores son responsables ni del contenido ni de las implicaciones de lo expresado en el artículo. 